\title{
Single-vortex-induced voltage steps in Josephson-junction arrays
}

\author{
P. H. E. Tiesinga ${ }^{1}$, T. J. Hagenaars ${ }^{1,3}$, J. E. van Himbergen ${ }^{1}$ and Jorge V. José ${ }^{2}$ \\ ${ }^{1}$ Instituut voor Theoretische Fysica, \\ Princetonplein 5, Postbus 80006, 3508 TA Utrecht, The Netherlands \\ ${ }^{2}$ Department of Physics and Center for Interdisciplinary Research on Complex Systems, Northeastern University, \\ Boston Massachusetts 02115, USA \\ 3 Institut für Theoretische Physik, Universität Würzburg, \\ Am Hubland, 97074 Würzburg, Germany
}

\begin{abstract}
We have numerically and analytically studied ac + dc driven Josephson-junction arrays with a single vortex or with a single vortex-antivortex pair present. We find single-vortex steps in the voltage versus current characteristics $(I-V)$ of the array. They correspond microscopically to a single vortex phaselocked to move a fixed number of plaquettes per period of the ac driving current. In underdamped arrays we find vortex motion period doubling on the steps. We observe subharmonic steps in both underdamped and overdamped arrays. We successfully compare these results with a phenomenological model of vortex motion with a nonlinear viscosity. The $I-V$ of an array with a vortex-antivortex pair displays fractional voltage steps. A possible connection of these results to present day experiments is also discussed.
\end{abstract}

PACS numbers: 74.50.+r, 74.60.Ge, 74.60.Jg

\section{INTRODUCTION}

The presence of giant Shapiro steps and giant fractional Shapiro steps in the $I-V$ characteristics of $2-\mathrm{D}$ Josephson-junction arrays has attracted significant attention recently [1]. These 2-D arrays may be of use as a source of coherent microwave radiation [8]. In a separate context the flux-flow dynamics of vortices has been studied [9 11]. The reported experimental observation of ballistic vortex motion [12 has also stimulated further theoretical and numerical investigations 13 17] of the mass and friction of a vortex in an array. Until now these numerical investigations have focused on dc driven vortices. In this work we perform numerical simulations on Josephson-junction arrays, with only one vortex or with a vortex-antivortex pair present in it, driven by a time-dependent current $i(t)=i_{d c}+i_{a c} \cos (2 \pi \nu t)$. We calculate the voltage $V$ versus $i_{d c}$ characteristics $(I-V)$. We find harmonic and subharmonic single-vortex voltage steps and analyze the underlying phase-locked vortex motion. A vortex-antivortex pair separated by a distance $\Delta x$ along the direction of the injected external current phase-locks on to fractional voltage steps.

The arrays are 2-D lattices of superconducting islands (sites) connected by Josephson junctions (bonds). The unit cells (plaquettes) of these lattices can be, for example, square or triangular. The vortices are represented by eddy-current patterns about a plaquette. Here we consider the classical regime defined by $E_{J} \gg E_{c}=e^{2} / 2 C$, where $E_{J}$ is the Josephson coupling energy and $E_{c}$ the charging energy of two islands, $e$ the electron charge, and $C$ the capacitance of a junction. In this regime quantum fluctuations are neglected, leaving the phases $\theta(\boldsymbol{r})$ of the Ginzburg-Landau order parameter on the islands as the only dynamical variables.

In this case the array is well-modeled by the Resistively Capacitively Shunted Junction (RCSJ) model, defined by the total bond current $i\left(\boldsymbol{r}, \boldsymbol{r}^{\prime}\right)$ between nearest neighbor sites $\boldsymbol{r}$ and $\boldsymbol{r}^{\prime}$

$$
\begin{aligned}
i\left(\boldsymbol{r}, \boldsymbol{r}^{\prime}\right) & =\beta_{c} \ddot{\theta}\left(\boldsymbol{r}, \boldsymbol{r}^{\prime}\right) \\
& +\dot{\theta}\left(\boldsymbol{r}, \boldsymbol{r}^{\prime}\right)+\sin \left[\theta\left(\boldsymbol{r}, \boldsymbol{r}^{\prime}\right)-2 \pi A\left(\boldsymbol{r}, \boldsymbol{r}^{\prime}\right)\right],
\end{aligned}
$$

plus Kirchhoff's current conservation conditions at each site. Here the dots represent time derivatives. The three contributions to $i\left(\boldsymbol{r}, \boldsymbol{r}^{\prime}\right)$ are the displacement, the dissipative and the superconducting currents, respectively. The phase difference across a junction is $\theta\left(\boldsymbol{r}, \boldsymbol{r}^{\prime}\right) \equiv \theta(\boldsymbol{r})-$ $\theta\left(\boldsymbol{r}^{\prime}\right)$. The currents are expressed in units of the junction critical current $I_{c}$; time is measured in units of the characteristic time $1 / \omega_{c}=\hbar /\left(2 e R_{n} I_{c}\right)$, and $\beta_{c}=\left(\omega_{c} / \omega_{p}\right)^{2}$ is the Stewart-McCumber parameter [20], with the plasma frequency $\omega_{p}$ defined as $\omega_{p}^{2}=2 e I_{c} / \hbar C$, and $R_{n}$ is the junction's normal state resistance. The bond frustration variable $A\left(\boldsymbol{r}, \boldsymbol{r}^{\prime}\right)$ is defined as the line integral of the vector potential $\boldsymbol{A}$ :

$$
A\left(\boldsymbol{r}, \boldsymbol{r}^{\prime}\right)=\frac{1}{\phi_{0}} \int_{\boldsymbol{r}}^{\boldsymbol{r}^{\prime}} \boldsymbol{A} \cdot d \boldsymbol{l},
$$

with the elementary quantum of flux $\phi_{0}=h / 2 e$. The frustration parameter $f$ measures the average flux piercing a plaquette, measured in units of $\phi_{0}$.

The motivation for this paper is twofold: to study the dynamics of a few vortices in an array, and to see whether the results can be generalized in order to explain dynamical non-equilibrium states, like the axisymmetric coherent vortex state [6]. Here we deal mainly with the analysis of single-vortex voltage steps. The motion of a vortex produces a Faraday voltage across the array. In this paper we find three types of new steps. First we find single-vortex voltage steps. The voltage $V$ on these steps 
is proportional to an integer multiple $n$ of the frequency of the ac drive:

$$
V=\frac{n h \nu}{2 e} \quad n=0,1,2, \cdot \cdot
$$

In the following we will also consider natural units $\hbar / 2 e=$ 1 . Then the voltage is normalized such that it corresponds to $2 \pi$ times the number of jumps between plaquettes of the vortex per time unit. This differs from another often used normalization by a factor $N_{y}$, the number of junctions perpendicular to the direction of the current injection. On such a step the motion of the single vortex is phase-locked to move an integer number of plaquettes per period $\frac{1}{\nu}$. Next we find subharmonic single-vortex steps in the $I-V$ 's of both overdamped and underdamped arrays. On these steps the voltage is:

$$
V=\frac{n}{m} \frac{h \nu}{2 e} \quad \begin{aligned}
n & =0,1,2, \cdots \\
m & =1,2, \ldots
\end{aligned}
$$

The dynamics on these steps corresponds to the vortex moving $n$ plaquettes in $m$ periods.

Finally we simulate a vortex-antivortex pair and obtain the following steps:

$$
\begin{aligned}
& V=\frac{n N_{y}}{m} \frac{h \nu}{2 e} \quad n=0,1,2, \cdot \\
& m=1,2, \cdot \cdot
\end{aligned}
$$

Each vortex moves $n N_{y} / 2$ plaquettes every $m$ periods. These new steps should be contrasted with other types of steps that arise in Josephson-junction arrays. In single junctions, harmonic $\left(V=n \frac{h \nu}{2 e}\right)$ voltage steps are present in both experimentally and numerically obtained $I-V$ 's [21. These steps are called single-junction Shapiro steps. On these steps the single junction has $n$ phase slips per period $\frac{1}{\nu}$. In simulations of and experiments on ac $+\mathrm{dc}$ driven arrays so-called giant Shapiro steps have been observed [1] 3]. On these steps the $N_{x} N_{y}$ individual junctions along the direction of the external current are all phase-locked on the same single junction Shapiro step, and one obtains

$$
V=N_{x} N_{y} \frac{n h \nu}{2 e} \quad n=0,1,2, \cdot \cdot
$$

The harmonic and subharmonic single-vortex voltage steps are therefore small in comparison to the giant Shapiro steps. Subharmonic giant Shapiro steps are observed experimentally [3] and in simulations [6]:7]. A particular example of these half-integer steps is found in the axisymmetric coherent vortex states (ACVS). These stationary states correspond to an oscillating pattern of vortex and antivortex streets, which arrange themselves at a well-defined angle with respect to the current direction [6].
In studies of and experiments on ac + dc driven arrays, with an average rational flux $\frac{p}{q} \phi_{0}$ piercing through each plaquette, giant fractional Shapiro steps were observed [2, 田5]. On these steps the voltage is:

$$
V=\frac{N_{x} N_{y}}{q} \frac{n h \nu}{2 e} \quad n=0,1,2, \cdots
$$

An explanation for such steps has been proposed and verified in simulations [2, n]: an $f=\frac{p}{q}$ array has $q$ degenerate ground states, consisting of vortex lattices, carried into each other by translations. In one period of the driving current the vortex lattice moves from one degenerate state to the next. After $q$ periods every vortex has moved through the whole array, generating the observed voltage. This shows that there are two kinds of Shapiro steps in Josephson-junction arrays. Those based on the coherent phase slips of all the individual junctions in the array (Giant Shapiro steps) and those involving coherent oscillatory vortex motions (giant fractional Shapiro steps and ACVS).

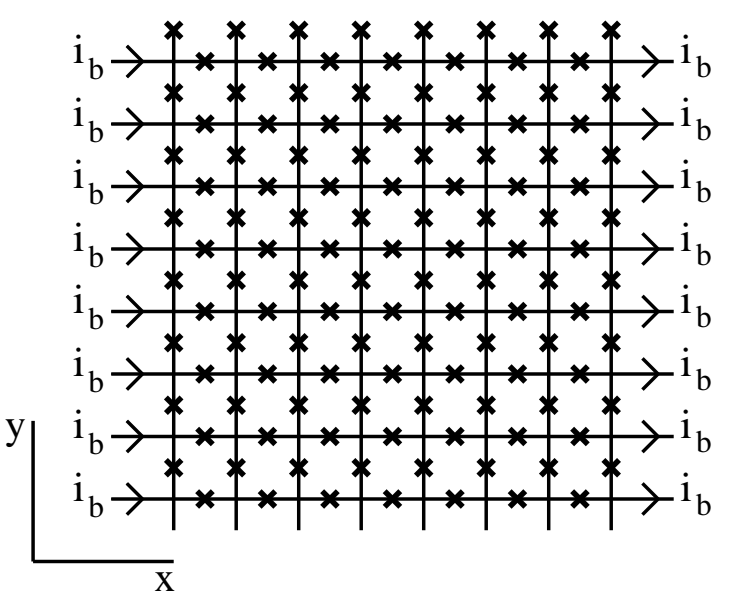

FIG. 1. Array geometry used in the simulations, illustrated with an $8 \times 8$ array. Junctions are denoted as crossed bonds. Periodic boundary conditions are imposed in the $y$-direction, while the current bias $i_{b}$ is applied along the $x$-direction.

The approach of our study is to have only one vortex, which makes it is possible to separate the effect generic of vortex motion from the effects of interaction between them. One can systematically study the underlying microscopic dynamics of the phase-locked vortex motion. Then by considering a vortex-antivortex pair one can study the effect of interaction in its most simple form on the phase-locked states. The vortex dynamics on singlevortex voltage steps has a number of new and interesting features, e.g. we observe vortex motion with period doubling in underdamped arrays. The underlying micro- 
scopic vortex motion repeats itself only after $2,4,8$, or even 16 periods $\frac{1}{\nu}$, although the vortex still moves, on average, a fixed number of plaquettes per period. In this paper we discuss how these steps can observed experimentally in arrays with a low density of vortices.

Previous authors [9,10,17 have studied simple models of vortex motion. In these models the vortex is described as a point particle of mass $M\left(\beta_{c}\right)$ experiencing a certain friction, and moving in a sinusoidal potential. In Ref. [17] we found that the $I-V$ of a de driven array in the vortex regime is well described by such a vortex equation of motion in terms of the vortex-coordinate $y$ containing, instead of the usual linear viscous force, a nonlinear one

$$
M\left(\beta_{c}\right) \ddot{y}+\frac{A\left(\beta_{c}\right) \dot{y}}{1+B\left(\beta_{c}\right)|\dot{y}|}+i_{0} \sin 2 \pi y=i
$$

In this equation $A\left(\beta_{c}\right)$ and $B\left(\beta_{c}\right)$ are phenomenological parameters. This analysis is similar in spirit to the description of long Josephson junctions in terms of the fluxon coordinates [18. An interesting question previously left unaddressed, and considered in this paper, is whether a phenomenological model for the vortex coordinate can still reproduce the $I-V$ of an ac + dc driven array. In this work we resolve this question and we compare the $I-V$ of an array with the result of Eq. (6) with a time-dependent current $i=i_{d c}+i_{a c} \cos 2 \pi \nu t$, and using the parameters $M\left(\beta_{c}\right), A\left(\beta_{c}\right)$ and $B\left(\beta_{c}\right)$ obtained for dc driven arrays. The results of Eq. (6) and the simulations are in reasonable agreement over a broad range of values of the frequency and the amplitude of the ac drive. In other words the vortex experiences a nonlinear friction in an ac+dc driven array. The outline of the paper is as follows. In section II we discuss the calculational algorithm to compute the $I-V$. In section III we present the $I-V$ 's containing the harmonic and subharmonic single-vortex steps and discuss the possibility of experimental verification. We then study subharmonic single-vortex voltage steps and period doubled vortex motion using the microscopic current distribution in the array as a function of time. Finally we compare the result of Eq. (6) to those of the $I-V$ 's obtained from the Josephson-junction array simulations. In section IV we investigate the effects of interaction and discuss the vortex-antivortex voltage steps in the $I-V$. In section $\mathrm{V}$ we present our conclusions.

\section{CALCULATIONAL APPROACH}

We numerically solve the equations of motion for a twodimensional array. We show the square lattice of $L_{x} \times L_{y}$ sites in Fig. 11. The sites are connected through Josephson junctions, denoted by crosses. We use the RCSJmodel of Eq. (1) to relate the current $i\left(\boldsymbol{r}, \boldsymbol{r}^{\prime}\right)$ through the Josephson junction to the phase difference $\theta\left(\boldsymbol{r}, \boldsymbol{r}^{\prime}\right)$.
We use periodic boundary conditions (PBC) in the $y$ direction, while the current is fed in and taken out along the $x$-direction. This set of coupled nonlinear differential equations can be integrated efficiently using a fast Fourier transform algorithm [6,22]. The array consists of $N_{x} \times N_{y}$ plaquettes $\left(N_{x}=L_{x}-1\right.$ and $\left.N_{y}=L_{y}\right)$. The vorticity $n(\boldsymbol{R})$ is defined as:

$$
2 \pi n(\boldsymbol{R})=2 \pi f+\sum_{\mathcal{P}(\boldsymbol{R})}\left(\theta\left(\boldsymbol{r}, \boldsymbol{r}^{\prime}\right)-2 \pi A\left(\boldsymbol{r}, \boldsymbol{r}^{\prime}\right)\right) .
$$

Here $\mathcal{P}(\boldsymbol{R})$ denotes an anti-clockwise sum around the plaquette $\boldsymbol{R}$ and the gauge-invariant phase difference $\theta\left(\boldsymbol{r}, \boldsymbol{r}^{\prime}\right)-2 \pi A\left(\boldsymbol{r}, \boldsymbol{r}^{\prime}\right)$ is taken between $-\pi$ and $+\pi$.

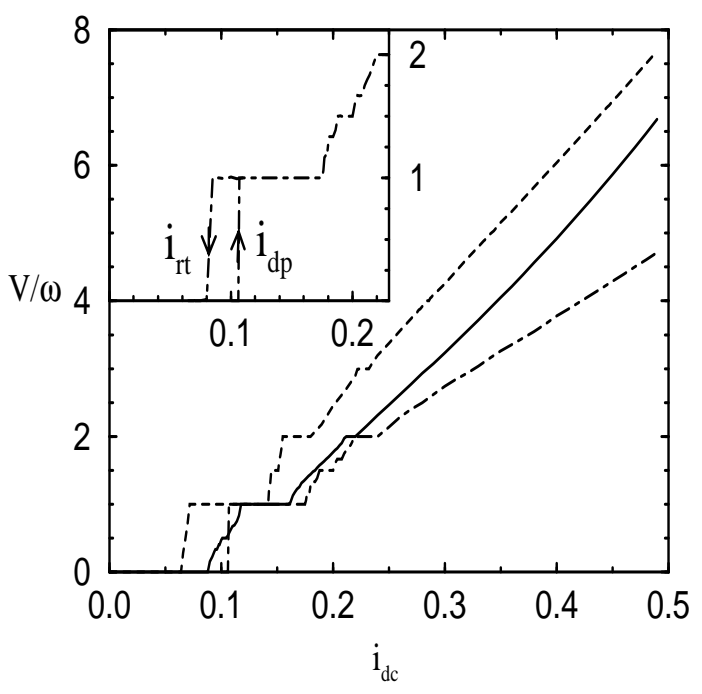

FIG. 2. $I$ - $V$ 's obtained from simulation of a $16 \times 32$ array with parameters, $i_{a c}=0.10, \nu=\frac{1}{25}$ and $\beta_{c}=0$ (continuous line); $\nu=\frac{1}{50}$ and $\beta_{c}=5$ (dotted line); and $\nu=\frac{1}{50}$ and $\beta_{c}=20$ (dashed-dotted line). The voltage in natural units is normalized by $\omega=2 \pi \nu$. The inset contains a close-up of the $\beta_{c}=20$ curve, with an added $I-V$ branch showing hysteresis.

We are interested in the behavior of vortices in Josephson-junction arrays. Stable vortices can be explicitly introduced in the initial phase-configuration by the application of a small frustration [17]. The plaquette coordinate $\boldsymbol{R}$ with unit vorticity will be called the topological vortex coordinate. The voltage $V(t)$ is obtained from:

$$
V(t)=\sum_{y=0}^{L_{y}-1} \frac{d}{d t}\left[\theta\left(L_{x}-1, y\right)-\theta(0, y)\right]
$$

The time average of $V(t)$ is related to the average vortex velocity $v=\frac{V}{2 \pi}$. The microscopic dynamics of the vortex motion is reflected in the eddy-current distribution $C(\boldsymbol{R}, t)$,

$$
C(\boldsymbol{R}, t)=\sum_{\mathcal{P}(\boldsymbol{R})} i\left(\boldsymbol{r}, \boldsymbol{r}^{\prime}, t\right)
$$


Where $\mathcal{P}(\boldsymbol{R})$ is the anti-clockwise sum over bonds about a dual lattice site $\boldsymbol{R}$. The vortex shows up as a local extremum in the eddy-current distribution.
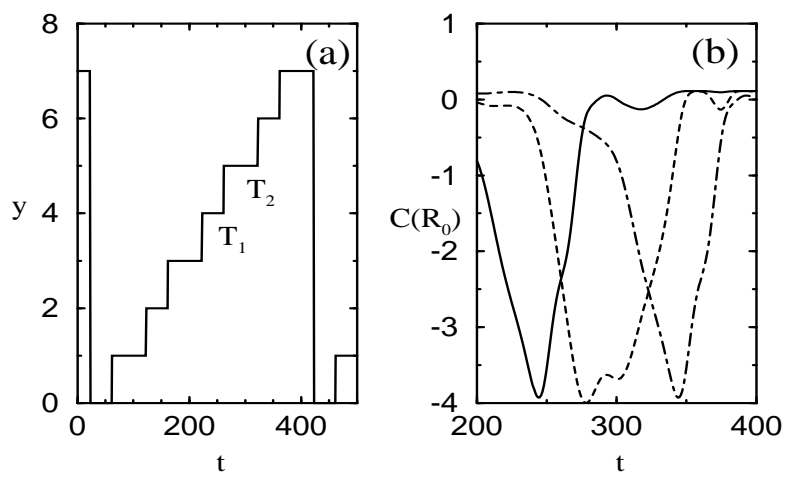

FIG. 3. Period doubling on the $n=1$ step. The results are obtained from a simulation of an $8 \times 8$ array, with parameters $\beta_{c}=25, \nu=1 / 50, i_{a c}=0.10$, and $i_{d c}=0.13$. In (a) we plot the vortex position versus time. In (b) we plot the eddy currents versus time at three adjacent plaquettes of the middle column. The continuous, dotted and dot-dashed lines are respectively the first, second and third plaquette in the middle column of the array. The minima in these graphs indicate the position of the vortex.

\section{SINGLE-VORTEX-INDUCED VOLTAGE STEPS}

In this section we present the results of our simulations of a single vortex in a Josephson-junction array. In the first subsection we discuss results for the $I-V$ characteristics. In the next subsection a detailed description of the single-vortex voltage steps is given. We discuss the microscopic vortex motion, including its period doubling and the subharmonic single-vortex voltage steps. This is followed by a comparison of a simple model for vortex motion to the simulation results, and finally the discussion of possible experimental verification.

\section{A. The $I-V$ 's}

The $I-V$ 's are generated by gradually increasing the bias current $i_{d c}$ from 0 starting with an initial configuration containing a vortex. We express the voltage in natural units $\frac{\hbar}{2 e} \equiv 1$ and normalized by the frequency $2 \pi \nu$. The harmonic steps then occur at integer voltage values.

For currents below the depinning current $i_{d p}$ the voltage is zero (see Fig. 2), when it is averaged over enough periods of the ac drive. The vortex deforms in response to the ac+dc drive, but stays in the same plaquette [23. Or, for low enough frequency $\nu$ and large enough $i_{a c}$, it can even oscillate back and forth over a finite number of plaquettes.

The second branch of the $I-V$ is generated by decreasing the bias current. As the initial phase configuration one uses the final phase configuration obtained in the upward sweep. At the retrapping current $i_{r t}$ the average voltage returns to zero. In underdamped arrays the retrapping current $i_{r t}$ can be different from $i_{d p}$. This hysteretic behavior has often been interpreted as evidence for inertia of the vortex in the dc driven case. An example of hysteresis is shown in the inset of Fig. 2. Focusing the discussion on the upward current sweep we encounter the first plateau at $V=2 \pi \nu$. This is the first single-vortex step. The vortex moves on average one plaquette per period. There are two more steps visible at multiples of $2 \pi \nu$ in Fig. 2. Above $i_{d c} \approx 0.25$ no steps are visible anymore. The step width has become smaller than the current grid size $\Delta i_{d c}=0.01$. The $\beta_{c}=0 I-V$ exhibits a pronounced upward curvature. Hence the vortex viscosity is nonlinear [17]. Between the $n=1$ and $n=2$ step a small subharmonic step, at $V / 2 \pi \nu=\frac{3}{2}$, is visible in Fig. 2. On this step the vortex moves three plaquettes every two periods. Using a smaller $\Delta i_{d c}=0.002$ one can even observe the $\frac{4}{3}$ and $\frac{5}{3}$ steps.

Increasing the damping parameter $\beta_{c}$ for given $\nu$, shifts the steps to higher values of the current bias $i_{d c}$. This is due to an increase in the vortex viscosity with $\beta_{c}$ [17. When $\beta_{c}$ is changed from 5 to 20 , the $n=3$ step width is gradually reduced to below the current grid size $\Delta i_{d c}=0.01$. For $\nu>1.0$ no integer steps are present any more. For these frequencies the velocity for which the vortex would phase lock on the first integer step lies above the maximum vortex velocity in the array.

The width of a particular step varies in an oscillatory fashion as a function of $\frac{i_{a c}}{2 \pi \nu}$. This is qualitatively similar to the step width behavior of a single junction, which varies as a Bessel function of $i_{a c}$. By adjusting $i_{a c}$ one can make more steps visible.

\section{B. Single-vortex-induced voltage steps}

We now turn to the microscopic vortex motion on the single-vortex voltage steps. We first consider the $n=0$ step, i.e. the response of a vortex that is pinned in one plaquette. One may expect the response to have the same period as the ac drive. In that case the quantity $v_{p}$, the voltage averaged over one period of the drive, is constant and equal to zero. We find, however, that $v_{p}$ can be nonzero on the $n=0$ step. This is repeated periodically in time. In table 1 we show the periodicity of $v_{p}$, (in units of the driving period) as a function of $i_{d c}$ for an $8 \times 8$ array with parameters $\beta_{c}=25, \nu=1 / 50, i_{a c}=0.1$.

Next we focus on the $n=1$ step in table The vortex depins at $i_{d c} \approx 0.12$. For $i_{d c}=0.13$ the voltage $v_{p}$ alternates between two different values. Figs. 3 and 4 show the corresponding vortex motion. Fig. 3(a) shows the 
topological vortex coordinate, defined in Eq. (7), versus time. It shows that the time the vortex spends at one plaquette alternates between two values $T_{1}$ and $T_{2}$. The sum $T_{1}+T_{2}$ is equal to $2 / \nu$. In Fig. $3($ (b) we plot the eddy currents of three adjacent plaquettes versus time. In Fig. A we show snapshots of the spatial distribution of eddy currents. Frames 0 and 10 are almost equivalent. A jump occurs between frames 2 and 3 and between frames 8 and 9 . The difference between $T_{1}$ (frames $0-2$ ) and $T_{2}$ (frames 3-8) is clearly visible. All the figures show that the actual vortex motion is periodic with twice the period of the driving force.

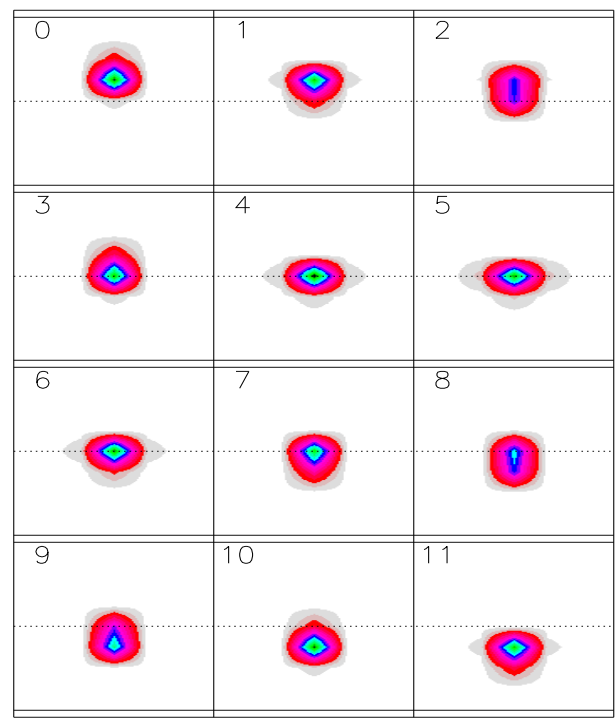

FIG. 4. Period doubling on the $n=1$ step. Parameters are the same as in Fig. 3. We show snapshots of the eddy current distributions at different times. The time step between two snapshots is 10 (in units of $1 / \omega_{c}$ ). The eddy-current distributions are smoothed out by interpolation.

Subharmonic steps turn out to be a generic feature of the $I-V$ 's of ac+dc driven arrays. They are observed for different values of $i_{a c}, \nu, \beta_{c}$ and system sizes. Many different voltage values are possible. We studied a $\frac{1}{2}$ step in detail. At $i_{d c}=0.11$ we found an $n=\frac{1}{2}$ step in a $\beta_{c}=0, \nu=1 / 25,8 \times 32$ array. On this step the generic behavior is as follows. Although the vortex deforms significantly during the first period, the topological vortex coordinate does not change. Only in the second period it jumps to the neighboring plaquette. We now describe a typical example.

In Fig. 5 we show the eddy current versus time of the plaquette containing the vortex. Both curves have two minima: $A$ and $B$. In minimum $B$ the vortex has a different shape (and hence feels a different potential) than in $A$. From this we can deduce the following scenario. During the first period the vortex deforms from configuration $A$ to $B$, staying in the same plaquette. In the following period it jumps to the next plaquette into the adjacent minimum $A$. We also found a case $\left(i_{d c}=0.072\right.$, $\nu=1 / 50$ ) in which the vortex briefly jumped to the next plaquette (and returned to the original plaquette) during the first period.

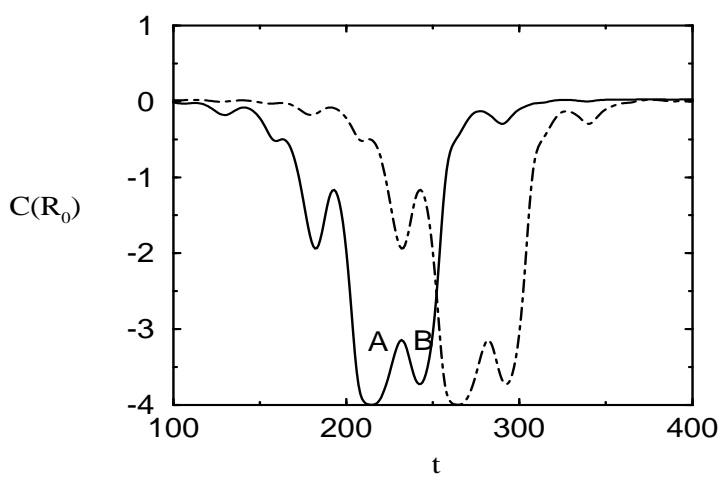

FIG. 5. Vortex motion on a $\frac{1}{2}$ step for an $8 \times 32$ array, with parameters $\beta_{c}=0, \nu=1 / 25, i_{a c}=0.10$, and $i_{d c}=0.11$. We plot the eddy current versus time, as in Fig. 3(b), for two adjacent plaquettes (denoted by the continuous and the dot-dashed line respectively). The minima $A$ and $B$ in this graph indicate the position of the vortex.

\section{Comparison to a phenomenological model of vortex motion}

In this subsection we compare the results of the numerical simulations to the results of a simple model for the vortex motion. The motion of a single vortex in an array can be modeled by that of a point particle with mass $M$ that, driven by a (Lorentz) force $i$, moves through a sinusoidal pinning potential and experiences a linear viscous damping force with constant viscosity coefficient $\eta$ [9,10]. The vortex mass $M$ can be calculated by equating the electromagnetic energy stored in the array to a vortex kinetic energy $\frac{1}{2} M \dot{y}^{2}$. The friction can be determined by equating the dissipated energy to $\eta \dot{y}^{2}$. This results in the following equation of motion for position $y(t)$, generalized to include a time dependent driving force $i$.

$$
\pi \beta_{c} \ddot{y}+\pi \dot{y}+i_{0} \sin 2 \pi y=i_{d c}+i_{a c} \cos 2 \pi \nu t .
$$

For a square array $i_{0} \approx 0.10$ [9]. Every time the particle moves one plaquette $(y \rightarrow y+1)$ an integrated $2 \pi$ voltage pulse is generated across the array in the $x$-direction. The average of $2 \pi \dot{y}$ is then the dimensionless average voltage measured across an array.

We recently introduced a modified vortex equation of motion [17] for dc driven vortices, including a nonlinear viscosity

$M\left(\beta_{c}\right) \ddot{y}+\frac{A\left(\beta_{c}\right) \dot{y}}{1+B\left(\beta_{c}\right)|\dot{y}|}+i_{0} \sin 2 \pi y=i_{d c}+i_{a c} \cos 2 \pi \nu t$. 
The parameter values for overdamped case are $M\left(\beta_{c}\right)=$ $0, A \approx 2.7, B \approx 1.8, i_{0}=0.1$ [17].

Equations (10) and (11) are similar to the equations describing single Josephson junctions. To connect to the single-Josephson-junction literature, replace $y$ by a phase $\theta=2 \pi y$, divide by $i_{0}$ and absorb the coefficient in front of the first derivative of $\theta$ in a new time unit:

$$
\beta \ddot{\theta}+\frac{\dot{\theta}}{1+\mathcal{B} \dot{\theta}}+\sin \theta=\bar{i}_{d c}+\bar{i}_{a c} \cos \Omega \tau .
$$

Where $\beta=M\left(\beta_{c}\right) \frac{i_{0} 2 \pi}{A^{2}}, \mathcal{B}=\frac{i_{0}}{A} B, \Omega=\nu \frac{A}{i_{0}}, \bar{i}_{a c}=\frac{i_{a c}}{i_{0}}$ and $\bar{i}_{d c}=\frac{i_{d c}}{i_{0}}$.

To allow for a more quantitative comparison we use the procedure described in Ref. [17] to find the parameters $A$ and $B$ in Eq. (11). That is we fit the $I-V$ curve in the dc driven case to the form

$$
\frac{\sqrt{i_{d c}^{2}-i_{0}^{2}}}{A-B \sqrt{i_{d c}^{2}-i_{0}^{2}}}
$$

Given these parameters one can numerically calculate the $I-V$ predicted by Eq. (11). In Fig. 6 we compare these results for one specific $i_{a c}, \nu$ and $\beta_{c}$ to the $I-V$ obtained from simulations of the full array. The results of Eq. (11) are in good agreement with the simulation for larger currents $i_{d c}>0.3$. For this current regime the steps are too small with respect to the current grid $\Delta i_{d c}=0.01$ to be visible.

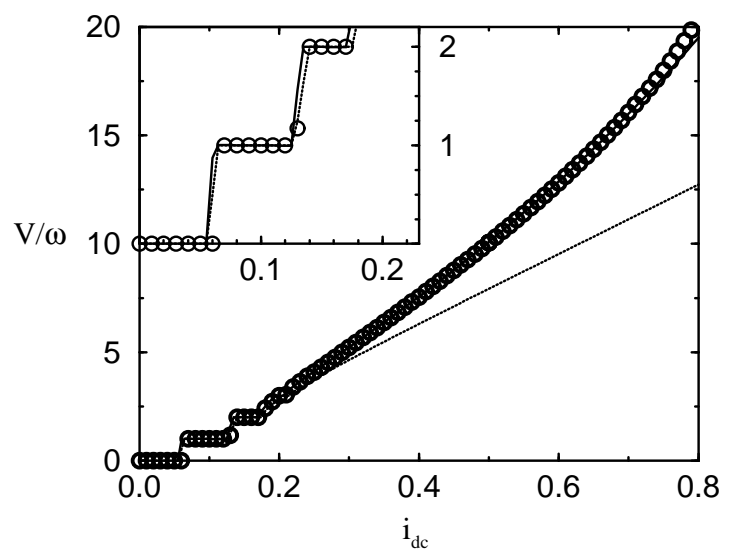

FIG. 6. Comparison of results the model Eq. (12) (dotted line, parameters: $\Omega=\pi / 5, \beta=0.4$, and $\left.\bar{i}_{a c}=1.0\right)$ and (full line, parameters: $\Omega=0.617, \mathcal{B}=0.0424, \beta=0$, and $\bar{i}_{a c}=1.0$ ) to the simulations of the full $16 \times 32$ array (circles, $\nu=\frac{1}{50}, \beta_{c}=2$, and $\left.i_{a c}=0.10\right)$.

For lower currents the agreement is less satisfactory. The steps in the simulations and in Eq. (11) do overlap to a large extent, but the lower edge of the steps is underestimated by Eq. (11), especially at depinning. However one finds large deviations when comparing the simulation data to Eq. (10), the model with linear viscosity. The phenomenological vortex mass for $\beta_{c}<35$ is $M\left(\beta_{c}\right)=0$, as found from the dc $I-V$ curves in Ref. [17]. In the dc+ac case, however, we find small hysteresis loops in the $I-V$. One could interpret this as an indication for the presence of a non-zero $M\left(\beta_{c}\right)$. The hysteretic behavior of Eq. (11) is complicated. Using different $M \neq 0$ with the computed values of $A\left(\beta_{c}\right)$ and $B\left(\beta_{c}\right)$ did not yield better agreement with the simulation results.

\section{Experimental verification}

In order to see how the single vortex phase-locking mechanism would manifest itself in experimentally accessible conditions, we now reinstate physical dimensions using experimental parameters 24]. Typical values for the parameters are $I_{c}=0.01-2.0 \mu \mathrm{A}, I_{c} R_{n}=300 \mu \mathrm{V}$, $\omega_{c}=\frac{2 e R_{n} I_{c}}{\hbar} \sim 1 \mathrm{GHz}$ and $\beta_{c}=10-100$. The singlevortex voltage steps would occur for $i_{d c}=0.10-0.30 I_{c}$ and for frequencies $\nu<0.10 \cdot \omega_{c} \sim 100 \mathrm{Mhz}$, and $\beta_{c}<50$. Experiments are conducted at a finite temperature. Voltage steps are observed as a reduction in the differential resistance $\frac{d V}{d I}$, while at zero temperature $\frac{d V}{d I}=0$ on a step. This reduction should be large enough, and extend over a sufficiently large current range, in order to be measurable. The temperature is expressed in units of $T_{0}=\frac{\hbar I_{c}}{2 e k_{B}} \approx 2 \times 10^{7} I_{c}$. We have performed a $T \neq 0$ simulation for one particular case: a $16 \times 16$ array with parameters $\nu=\frac{1}{25}, \beta_{c}=0$, and $i_{a c}=0.10$. The simulation was done using the algorithm introduced in Ref. [25], as extended for arrays [6]. We found that up to $T=0.004 T_{0}$ the $n=1$ step was clearly visible. For $T=0.008 T_{0}$ a reduction in $\frac{d V}{d I}$ was hardly discernible. This puts an upper bound on the appropriate temperatures, varying from $T=1$ to $200 \mathrm{mK}$ with the value of $I_{c}$.

To study the single-vortex phase-locking phenomena experimentally, one can use relatively small samples, to make sure that only a small absolute number of vortices is present at any time, or a large array with a low vortex density. In the former type of experiments the interaction of the vortex with the boundaries, or equivalently with image antivortices, has to be taken into account. When moving to a boundary, a vortex may either escape from the array or reflect as an antivortex [19]. In the parameter regime studied in this paper, vortices are entering the array and leaving it again at the opposite boundary. In practice the small number of vortices present will therefore fluctuate slightly. This may effect the way in which phase-locking is established as compared to our simulation in which only one vortex is present at any time. To estimate this effect we have simulated a finite array in a small magnetic field and also found steps in the I-V's due to phase-locking with the ac drive at voltages slightly different from those mentioned above. 
We also have performed simulations of triangular arrays, often used experimentally, and find qualitatively the same scenario for the occurrence of single-vortex voltage steps, as reported in section IIIA.

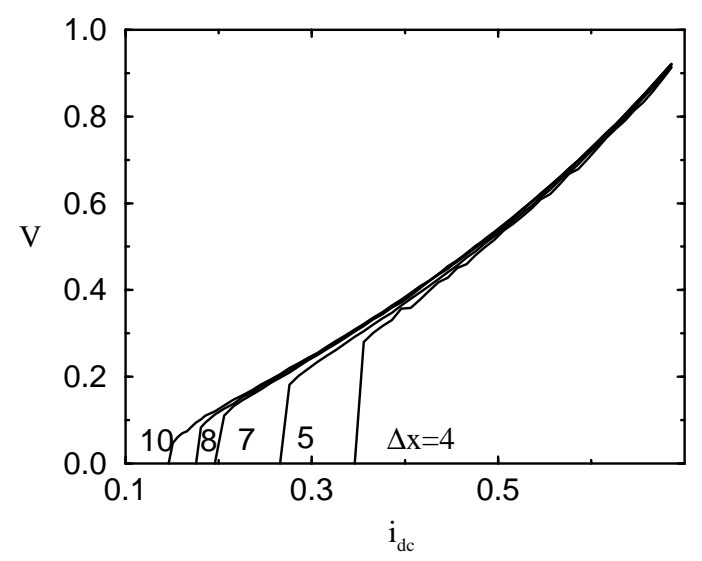

FIG. 7. $I-V$ of a vortex and an antivortex separated by a distance $\Delta x=4,5,7,8$, and 10 in an overdamped dc driven $32 \times 32$ array. The annihilation current is the current at which the average voltage drops to zero in the $I-V$ of the downward current sweep.

\section{VORTEX-ANTIVORTEX PAIR-INDUCED STEPS}

In this section we consider the effect of vortexantivortex interaction on the single-vortex voltage steps. In the first subsection we show the fractional voltage steps in the $I$ - $V$ due to the vortex-antivortex interaction, and obtain these steps from a phenomenological model for vortex motion including the logarithmic interaction. In the second subsection we briefly discuss the analogous behavior of excess and missing vortices in a checkerboard groundstate of an $f=\frac{1}{2}$ array.

\section{A. Vortex-antivortex pair in an $f=0$ array}

We calculate the zero temperature $I-V$ 's of an array containing a vortex-antivortex pair. There is no applied magnetic field, and the pair is included in the initial configuration by construction [17. In order to show how one may obtain meaningful results starting from such a metastable configuration, let us first discuss the case of only a dc drive. When the applied current is zero, the only mechanism that may prevent the annihilation of the pair is the pinning of the lattice. Pinning will prevent annihilation if the mutual separation is at least 8 lattice constants. This minimum distance is in approximate agreement with the value of 0.10 [9] (in units of the junction critical current) for the maximum pinning force on a vortex in an infinite system. However, when the current is non-zero, and the vortices move in different rows (in opposite directions), annihilation may be absent even when the perpendicular distance between the rows is less than 8 lattice constants, depending on the magnitude of the current. We have studied this stabilization by the driving force quantitatively by performing downward current sweeps starting from a number of initial configurations containing pairs with different separation distances $\Delta x$. The $I-V$ 's are shown in Fig. 7. When the pair is unstable, and thus annihilated, the average voltage obtained from the simulations sharply drops to zero. This annihilation current strongly depends on the separation distance of the pair in the initial configuration. A higher current is needed to stabilize a smaller pair.

Next we consider the behavior of an ac+dc driven pair. Since two vortices move in opposite directions under the influence of the current, their mutual distance will change in time. Phase-locking can therefore not be established for a voltage corresponding to one jump per period, since in the next period the environment has changed, and thereby the interaction strength. We nevertheless find steps in the $I-V$ of these systems. Once the vortex and the antivortex have traversed $L_{y} / 2$ plaquettes, they cross each other again because we have periodic boundary conditions. Phase-locking is established if at that point the phases are the same again. If phase-lock is established over $n$ traversals, and the motion is periodic with period $m$ (in units of the external frequency $\nu$ ), then the observed voltage is: $V=n L_{y} / m 2 \pi \nu$. We can find each of these steps in the I-V in Fig. 8, although for higher $n$ the step width decreases rapidly. The step width is typically small $(\sim 0.01)$.

When the vortex and antivortex are separated by a large distance, one would expect to see single-vortex voltage steps again. This does not occur for the system size we considered due to the long range of the interaction. In section IIIB we considered models for a single vortex, here we consider a model for a pair. We model the vortexantivortex interaction as being logarithmic. In addition we have to take into account the periodic boundary condition along the $y$-direction: the vortex can see an antivortex behind it and in front of it (and vice versa). We denote the position of the vortex by $y_{1}$, and that of the antivortex by $y_{2}$. Let the coordinates take the values $0 \leq y_{i}<L_{y}$. The images are then at $y_{i} \pm L_{y}$. We only take the direct interaction and the interaction of the nearest image charge into account (i.e. at a distance less then $L_{y}$ ). The only stable current-driven pairs in the simulation are the ones that move at a fixed separation $\Delta x$, we therefore fixed the separation $\Delta x$ in this model. 
This leads to the following equation of motion:

$$
\begin{aligned}
\eta\left(\dot{y}_{1}\right) \dot{y}_{1}=+i(t)-i_{d} \sin \left(2 \pi y_{1}\right) & -\frac{y_{1}-y_{2}}{\Delta x^{2}+\left(y_{1}-y_{2}\right)^{2}} \\
& +\epsilon \frac{L_{y}-\left|y_{1}-y_{2}\right|}{\Delta x^{2}+\left(L_{y}-\left|y_{1}-y_{2}\right|\right)^{2}} \\
\eta\left(\dot{y}_{2}\right) \dot{y}_{2}=-i(t)-i_{d} \sin \left(2 \pi y_{2}\right) & +\frac{y_{1}-y_{2}}{\Delta x^{2}+\left(y_{1}-y_{2}\right)^{2}}+ \\
& -\epsilon \frac{L_{y}-\left|y_{1}-y_{2}\right|}{\Delta x^{2}+\left(L_{y}-\left|y_{1}-y_{2}\right|\right)^{2}}
\end{aligned}
$$

Here $\epsilon$ is the sign of $y_{1}-y_{2}$. We have computed the $I$ $V$ 's using these equations and we indeed found steps at $V=n L_{y} / m 2 \pi \nu$. To capture the mechanism for the pair steps in the model, we found the essential ingredients to be the interaction and the restriction of $y_{i}$ to $L_{y}$, that is periodic boundary conditions in the $y$-direction. The detailed form of the interaction determines the bias currents for which the steps occur.

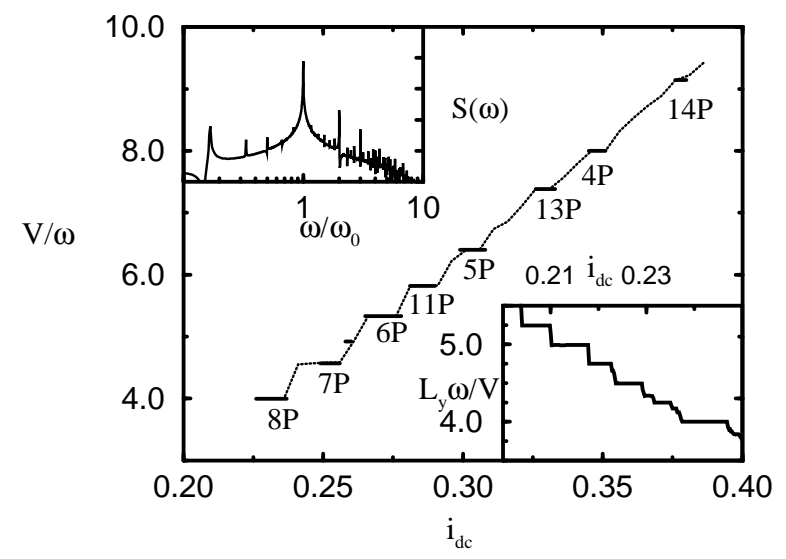

FIG. 8. $I-V$ of a vortex-antivortex pair with separation distance $\Delta x=7$ in an overdamped $32 \times 32$ array. The ac component of the driving current has frequency $\nu=\frac{1}{25}$ and amplitude $i_{a c}=0.10$. The labels $\mathrm{mP}$ signify the underlying periodicity of the motion on these steps (i.e. the $m$ in Eq. (5)). In the upper inset we show the spectral function of the voltage for the $6 \mathrm{P}$ step. In the lower inset we show the $I-V$ obtained from the model for vortex-antivortex pair motion in the text. We used the following parameters: $L_{y}=32,2 \pi \nu=1.186$, $\mathcal{B}=0.0674$, and $\Delta x=8$. Here we plot, instead of $V, L_{x} \omega / V$. Steps then arise at the values $\frac{m}{n}$. The higher $n$ the smaller the step width.

\section{B. Defects in fully frustrated $f=\frac{1}{2}$ array}

It was found in 13, 17 that the $I-V$ of a single excess vortex in a dc driven $f=\frac{1}{2}$ array resembles that of single vortex in the $f=0$ case up to a current $i_{d c}=0.34$. At the latter current the entire checkerboard of vortices depins, producing a voltage that is much larger than the single vortex response. The excess vortex moves in an even more nonlinear viscous fashion compared to $f=0$ [17.

Now we include an ac component to the dc driving current, and we find steps at $V=2 n(2 \pi \nu)$. The factor two can be explained using the scenario proposed in [28]: first the excess vortex pushes the vortex in front of it to jump, and then follows suit. In total two jumps have been made. Small subharmonic $V=n(2 \pi \nu)(n=1,3)$ steps can be distinguished in the $I-V$ shown in the inset of Fig. 9 .

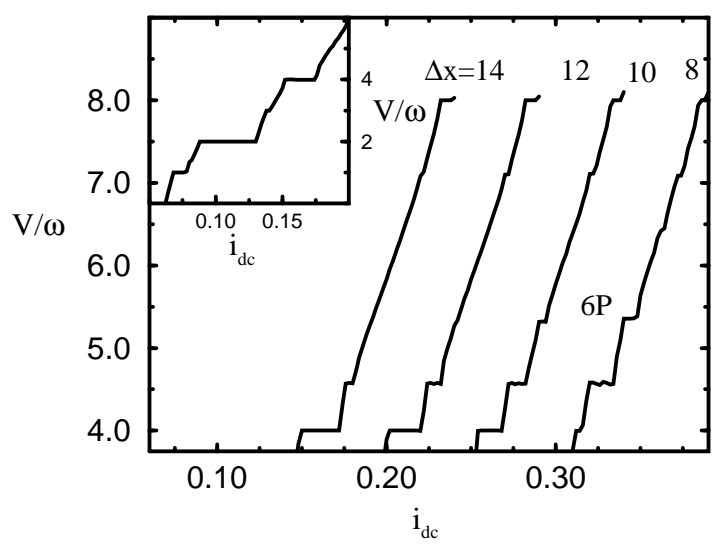

FIG. 9. $I-V$ obtained by driving an excess and a missing vortex, separated by $\Delta x$, in the vortex lattice of an overdamped fully frustrated $32 \times 32$ array. The ac component has frequency $\nu=\frac{1}{25}$ and amplitude $i_{a c}=0.10$. The curves are plotted for separations $\Delta x=8,10,12,14$. In the inset we show an $I-V$ of an extra vortex moving in the groundstate vortex lattice of the fully frustrated $16 \times 16$ array. The frustration is $f=\frac{1}{2}+\frac{1}{15 \times 16}, i_{a c}=0.10, \nu=\frac{1}{50}$, and $\beta_{c}=0$.

We find that the $I-V$ of an excess vortex and a missing vortex (with respect to the checkerboard vortex lattice groundstate) in the $f=\frac{1}{2}$ case is similar to that of a vortex and an antivortex respectively in the $f=0$ case. An excess vortex can be annihilated by a missing vortex. We calculated the $I-V$ for different values of $\Delta x$. In these $I$ $V$ 's one again finds the fractional vortex-antivortex-pair steps as in the $f=0$ case. One observes in Fig. 9 that as the separation $\Delta x$ becomes larger, these steps disappear, and the steps corresponding to single vortices grow, as one would intuitively expect. Finally only the latter remain.

\section{SUMMARY AND DISCUSSION}

In this paper we discussed the phase-locking behavior of a single vortex and a vortex-antivortex-pair under the 
influence of an ac $+\mathrm{dc}$ drive. We obtained harmonic and subharmonic single-vortex voltage steps, and studied the microscopic vortex dynamics. We found period doubled vortex motion. The $n=1$ step corresponds to a vortex jumping one plaquette per period, since in a periodic array all the plaquettes in the same column along the periodic direction are equivalent, and one would expect period one behavior. The simulation, in which period doubling vortex motion is observed, was performed in an $8 \times 8$ array with $\beta_{c}=25$. In this case the vortex motion can excite spin waves 13, 14. These spin waves cause a spatially modulated environment for the vortex. Similar phase-locking is observed in a ring of Josephson junctions 27.

The spin waves may also be responsible for the complicated behavior of the hysteresis we observed in ac $+\mathrm{dc}$ driven arrays as a function of $\beta_{c}$. In recent experiments subharmonic giant Shapiro steps [3] were observed in over- and underdamped arrays. In simulations it was shown that one can have such steps in a 2-D array by either including disorder [6] or inductive effects [7], or generally by any mechanism that breaks the translational invariance of the array. In overdamped single junctions one can only get subharmonics by using non-sinusoidal current-phase relationships [26]. Our simulations show that a single vortex itself may phase-lock on subharmonic steps. The simulation of the pair case shows that singlevortex voltage steps are replaced by fractional steps, which can be understood in terms of vortex interaction. Unfortunately this insight also makes it manifest why generalization to the dynamics associated with Shapiro steps that involve many vortices (such as the axisymmetric coherent vortex states) is not feasible. Another consequence of the fact that we find subharmonic steps, is that a more realistic vortex equation of motion (than Eq. (10) and (11)) should contain higher harmonic corrections to the sinusoidal potential. The presence of higher harmonics in the potential experienced by vortices was already noted by Lobb et al. [9].

We have shown that the parameters for which singlevortex voltage steps can be observed are within the reach of present day experiments.

\section{ACKNOWLEDGEMENTS}

We thank D. Domínguez, U. Geigenmüller, A. van Otterlo and A. van Oudenaarden for discussions. This work was supported in part by the Dutch organization for fundamental research (FOM). The work of JVJ has been partially supported by NSF grant No. DMR-9521845. TJH thanks the Bavarian "FORSUPRA" program for financial support.
[1] T.D Clark, Phys. Rev. B 8, 137 (1973); Ch. Leeman, Ph. Lerch and P. Martinoli, Physica (Amsterdam) 126B, 475 (1984).

[2] S.P. Benz, M.S. Rzchowski, M. Tinkham and C.J. Lobb, Phys. Rev. Lett. 64, 693 (1990).

[3] H.C. Lee, R.S. Newrock, D.B. Mast, S.E. Hebboul, J.C. Garland, C.J. Lobb, Phys. Rev. B 44921 (1991); S.E. Hebboul and J.C. Garland, Phys. Rev. B 4313703 (1991).

[4] K.H. Lee, D. Stroud, and J.S. Chung, Phys. Rev. Lett 64962 (1990); J.U. Free, S.P. Benz, M.S. Rzchowski, M. Tinkham and C.J. Lobb, Phys. Rev. B 417267 (1990); K.H. Lee and D. Stroud, Phys. Rev. B 43, 5280 (1991); M. Octavio, J.U. Free, S.P. Benz, R.S. Newrock, D.B. Mast and C.J. Lobb, Phys. Rev. B 444601 (1991).

[5] W. Yu, E.B. Harris, S.E Hebboul, J.C. Garland and D. Stroud, Phys. Rev. B 4512624 (1992).

[6] D. Domínguez, J.V. José, A. Karma, and C. Wiecko, Phys. Rev. Lett. 67, 2367 (1991); D. Domínguez, J.V. José, Phys. Rev. B 48, 13717 (1993).

[7] D. Domínguez, J.V. José, Phys. Rev. Lett. 69, 514 (1992); J.R. Phillips, H.S.J. van der Zant, J. White and T.P. Orlando, Phys. Rev. B. 50, 9387 (1994).

[8] S.P. Benz and C.J. Burrough, Appl. Phys. Lett. 58, 2162 (1991)

[9] C.J. Lobb, D.W. Abraham, and M. Tinkham, Phys. Rev. B 27, 150 (1983).

[10] U. Eckern and A. Schmid, Phys. Rev. B 39, 6441 (1989); U. Eckern, in Applications of Statistical and Field Theory Methods to Condensed Matter, Vol 218 of NATO Advanced Studies Institute,Series B: Physics, edited by D. Baeriswyl et al. (Plenum, New York, 1990), p. 311; M.S. Rzchowski, S.P. Benz, M. Tinkham, and C.J. Lobb, Phys. Rev. B 42, 2041 (1990); T.P. Orlando, J.E. Mooij, and H.S.J. van der Zant, Phys. Rev. B 43, 10218 (1991); U. Eckern and E.B. Sonin, Phys. Rev. B 47, 505 (1993).

[11] H.S.J. van der Zant, F.C. Fritschy, T.P. Orlando, and J.E. Mooij, Physica (Amsterdam) 165\&166B, 969 (1990); and H.S.J. van der Zant, C.J. Muller, L.J. Geerligs, C.J.P.M. Harmans, and J.E. Mooij, Phys. Rev. B. 38, 5154 (1988); H.S.J. van der Zant, F.C. Fritschy, T.P. Orlando, and J.E. Mooij, Phys. Rev. Lett. 66, 2531 (1991); and Phys. Rev. B 47, 295 (1993); T.S. Tighe, A.T. Johnson, and M. Tinkham, Phys. Rev. B 44, 10286 (1991);

[12] H.S.J. van der Zant, F.C. Fritschy, T.P. Orlando, and J.E. Mooij, Europhys. Lett. 18, 343 (1992).

[13] P.A. Bobbert, Phys. Rev. B 45, 7540 (1992); U. Geigenmüller, C.J. Lobb, and C.B. Whan, Phys. Rev. B 47, 348 (1993); W. Yu and D. Stroud, Phys. Rev. B 49, 6174 (1994).

[14] U. Geigenmüller, "Ballistic vortex motion in Josephson arrays", preprint.

[15] W. Yu, and D. Stroud, Phys. Rev. B 46, 14005 (1992).

[16] W. Yu, K.H. Lee, and D. Stroud, Phys. Rev. B 47, 5906(1993).

[17] T.J. Hagenaars, P.H.E. Tiesinga, J.E. van Himbergen and J.V. José, Phys. Rev. B 50, 1143 (1994); T.J. Hagenaars, P.H.E. Tiesinga, J.E. van Himbergen and J.V. José, Quantum Dynamics of Submicron Structures, Vol 291 of NATO Advanced Studies Institute, Series E: Ap- 
plied Sciences, edited by H.A. Cerdeira et al, 617 (1995).

[18] See for instance K.K. Likharev, Dynamics of Josephson Junctions and Circuits (Gordon and Breach, Philadelphia, 1986), and references therein.

[19] T.J. Hagenaars, J.E. van Himbergen, J.V. José and P.H.E. Tiesinga, Phys. Rev. B 53, 2719 (1996).

[20] D.E. McCumber, J. Appl. Phys. 39, 3113 (1968); W.C. Stewart, Appl. Phys. Lett. 12, 277 (1968).

[21] S. Shapiro, Phys. Rev. Lett 11, 80 (1963).

[22] H. Eikmans and J.E. van Himbergen, Phys. Rev. B 41, 8927 (1990).

[23] H. Eikmans and J.E. van Himbergen, Phys. Rev. B 45, 10597 (1992).

[24] These parameters are for arrays fabricated at Delft Technical University by A. van Oudenaarden. The bias current $I_{d c}$ can be measured with accuracy $\Delta I=10^{-9} A$, the voltage $V$ with $\Delta V=10^{-7} V$. The feasible frequency range is $\nu=1-10 \mathrm{MHz}$.

[25] H.S. Greenside and E. Helfand, Bell Syst. Tech. J. 60, 1927 (1981).

[26] M.J. Renne and D. Polder, Rev. Phys. Appl 9, 25 (1974); H. Lübbig and H. Luther, Rev. Phys. Appl. 9, 29 (1974)

[27] H.S.J. van der Zant, T.P. Orlando, S. Watanabe and S.H. Strogatz, Phys. Rev. Lett. 74, 174 (1995).

[28] E.K.F. Dang and B.L. Györffy, Phys. Rev. B 47, 3290 (1993).

\begin{tabular}{|l||c|c|}
\hline \hline$I_{d c}$ & step & period \\
\hline \hline $0.0-0.06$ & $n=0$ & 1 \\
0.075 & & 2 \\
0.08 & & 4 \\
0.085 & & 8 \\
0.088 & & 16 \\
0.09 & & $\sim 24$ \\
0.095 & & 8 \\
0.10 & & 4 \\
$0.105,0.11$ & & 2 \\
0.115 & & 1 \\
\hline 0.125 & $n=1$ & 4 \\
$0.13-0.155$ & & 2 \\
$0.165-0.19$ & & 1 \\
\hline \hline 0
\end{tabular}

TABLE I. The periodicity of the voltage $v_{p}$ averaged over one period of the driving force as a function of $i_{d c}$. The data is obtained from simulations of an $8 \times 8$ array, with parameters $\beta_{c}=25, \nu=1 / 50$, and $i_{a c}=0.10$. 